\title{
Cloud-based Optical Characterization of Hard Disk Drive Recording Heads
}

\author{
Weerayuth Khunrattanasiri, ${ }^{1}$ Anchyza Yokpradit, ${ }^{1}$ \\ Teerawat Tongloy, ${ }^{2}$ and Siridech Boonsang ${ }^{1 *}$ \\ ${ }^{1}$ Department of Electrical Engineering, Faculty of Engineering, \\ King Mongkut's Institute of Technology Ladkrabang, \\ 1, Soi Chalong Krung 1, Chalong Krung Road, Ladkrabang, Bangkok 10520, Thailand \\ ${ }^{2}$ Center of Industrial Robots and Automation (CiRA), College of Advanced Manufacturing Innovation (AMI), \\ King Mongkut's Institute of Technology Ladkrabang, \\ 1, Soi Chalong Krung 1, Chalong Krung Road, Ladkrabang, Bangkok 10520, Thailand
}

(Received December 18, 2017; accepted June 5, 2018)

Keywords: micro-ellipsometry, complex refractive index, private cloud, MQTT, flying height

In the hard disk drive (HDD) industry, the measurement of flying height is crucial for the verification of the recording head air-bearing design. The flying performance of recording heads has to be measured and compared with the original design to assure the reliability of HDDs. Optical interferometry is normally used to measure the flying height of recording heads. The key parameter for setting up the measurement is the refractive index, whose values normally deviate depending on machine conditions during the manufacturing processes. The variation in refractive index cannot be identified during flying height measurement because there is no on-line technique for doing so. In this paper, we present a novel technique using inhouse designed micro-ellipsometry to measure the complex refractive index combined with data transfer to a private cloud network via the Internet of Things message queuing telemetry transport (IoT MQTT) protocol. Therefore, the measurement of the complex refractive index can be carried out for each recording head and we can improve the precision of the flying height measurement. In addition, the information about refractive index not only can be shared with a flying height tester to correct the variation in each recording head but also can be used to assist air-bearing designers in the instantaneous validation of their design.

\section{Introduction}

As the capacity of hard disk drives (HDDs) increases exponentially, the flying height of recording heads is expected to be as small as about $10 \mathrm{~nm}$ during their operation. To guarantee the reliability of HDDs for long-term operation, the flying performance of recording heads has to be measured to validate the original air-bearing design. The flying height measurement of recording heads is a key process in the validation of HDD production. The precision of flying height measurement has to be $0.05 \mathrm{~nm}$. The important input parameter for the flying height

*Corresponding author: e-mail: siridech.bo@kmitl.ac.th

https://doi.org/10.18494/SAM.2018.1850 
measurement is the complex refractive index $(n, k)$ of the surface of the recording heads. The conventional standard interferometric flying height tester (D6) is typically used to measure the distance between the hard disk header and media. It is a well-known HDD industrial standard equipment that provides both a highly accurate measurement and good repeatability.

In setting up the process, a D6 machine requires the recording head's refractive index $(n, k)$ as user input. $n$ is a refractive index that indicates the optical phase velocity and $k$ is an optical extinction coefficient. The obtained values are used as the input constants to determine the flying height of a hard disk reader. The D6 machine itself cannot quantify the $n, k$ value. However, the $n, k$ value can normally be measured by a standard industrial ellipsometer. With the limited spot size of a conventional industrial ellipsometer. The $n, k$ value of an individual slider on a head gimbal assembly (HGA) cannot be measured. An example of an HGA is shown in Fig. . $^{(1)}$ The measured $n, k$ values from the slider bars are used as the representative values for the entire production (more than $500 \mathrm{k}$ samples). However, the condition of the machine during HDD manufacturing (from slider bars to HGA forms) causes the $n, k$ value to change. Therefore, the flying height measurement in a D6 machine suffers considerably from this discrepancy. Leong et al. ${ }^{(2)}$ presented a technique of determining the optical constants $(n, k)$ on a spot of an individual HGA. Their technique improves the accuracy and repeatability of flying height measurements. However, the statistical distribution of real HGAs has a substantial effect on the performance of their system.

Instead of using a conventional ellipsometer to measure the $n, k$ value, the development of a specially designed micro-ellipsometer may provide flexible solutions for performing measurement at the HGA level. Feke et al. ${ }^{(3)}$ demonstrated a measurement system based on a back focal plane micro-ellipsometer. They showed the ellipsometric analysis of various materials with a relatively small measurement spot size. They used the Fourier plane, which is sometimes called the back focal plane. However, a mechanical phase shifting technique may cause additional errors in the measured values. These measurement errors are primarily contributed by the nonlinear behavior and hysteresis of a PZT transducer used for creating mechanical movement. This shortcoming has been mentioned in numerous reports. ${ }^{(4,5)}$ To avoid the errors that arise from the nonlinear behavior and hysteresis of the PZT phase shifter,

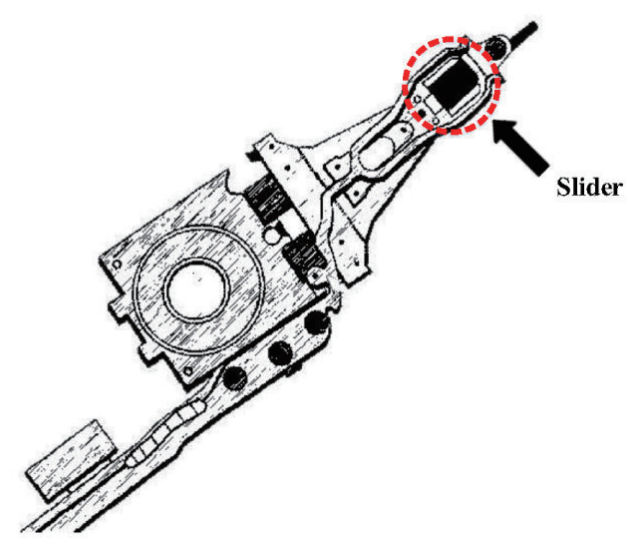

Fig. 1. (Color online) Example of a slider installed on an HGA. ${ }^{(1)}$ 
a system based on phase shifting interferometry has been developed. ${ }^{(6)}$ The optical phase of a light wave can be changed by modifying the polarization state of composite components of the original light wave. A rotating quarter-wave plate (QWP) was utilized to alter the light polarization state. It is normally located at the beam path where the reference and probe beams propagate collinearly after recombination. The rotation of a QWP can be precisely controlled. It is also less affected by the nonlinear behavior and hysteresis. Therefore, the high resolution in optical phase measurement can be achieved. In our system, we implement this technique to substitute a PZT phase shifter for optical phase measurement.

The complex refractive index of the surface of recording heads gives essential values for engineers to design and evaluate the performance of recording heads. These values are routinely used in several production departments and also different locations such as R\&D centers, a US headquarter, and a production line (D6 machine setting) in the manufacturing factory. Therefore, the cloud-based measurement system is a desirable solution for the instantaneous sharing of such information about the reflective index values. The push protocol is the most prevalent message transmission protocol for IoT devices. ${ }^{(7-10)}$ This is due to the fact that such a protocol is designed in the low-bandwidth network typically. Push message services implemented various protocol forms such as XMPP, CoAP, and MQTT. In particular, the MQTT protocol was considered to run on low-power devices efficiently as a light-weight protocol. It has been used in many IoT devices for instant messaging systems. The MQTT protocol is intended to ensure the quality of service $(\mathrm{QoS})$ of each short message. However, it could be challenging to ensure precise delivery of these messages when a number of them are transmitted from senders. The receiving order of the messages is essential for the production line since users' requirement is to trace $n, k$ information for each recording head. Therefore, in order to implement the MQTT messaging for distributing the measured reflective index values from our micro-ellipsometer machine (sender), the latency of data transmission should be carefully investigated.

In this research, we present the development of an optical characterization system for recording heads in a cloud-based environment. This system can measure the complex reflective index $(n, k)$ of an individual HGA online. We report a novel technique using micro-ellipsometry combined with phase shifting interferometry to measure the complex reflective index at a relatively small spot size $(0.1 \mathrm{~mm})$. The micro-ellipsometer utilizes the special properties of a high-numerical-aperture (NA) optical objective lens. The change in optical phase shift at different incident angles can be measured instantly using a high-NA objective lens. The phase shifting technique based on a rotating QWP is used to extract the optical phase shifts of the sand p-components of rays reflected from a high-NA objective lens. Our micro-ellipsometry system enables measurement over a relatively small spot size. The spot size is typically smaller than $0.1 \mathrm{~mm}$. The $n, k$ value is distributed from the micro-ellipsometer to a private cloud network. The private cloud utilizes the message queuing telemetry transport (MQTT) protocol for data distribution to related users. Our system offers the actual values measured for an individual slider on an HGA. As a result, the flying height measurement of the D6 machine is more precise. 


\section{System Descriptions}

\subsection{Micro-ellipsometer system configuration}

The diagram of the micro-ellipsometer is shown in Fig. 2. The photograph of the actual hardware employed in this research is shown in Fig. 3. An unpolarized beam of the $\mathrm{He}-\mathrm{Ne}$ laser is used as the light source of the measurement system. The wavelength of a He-Ne laser is $632.8 \mathrm{~nm}$. The unpolarized beam is converted into a polarized beam by polarizer $\mathrm{P}_{1}$, then the polarized beam is expanded by beam expander $\mathrm{BX}_{1}$. The beam is directed through a high-NA

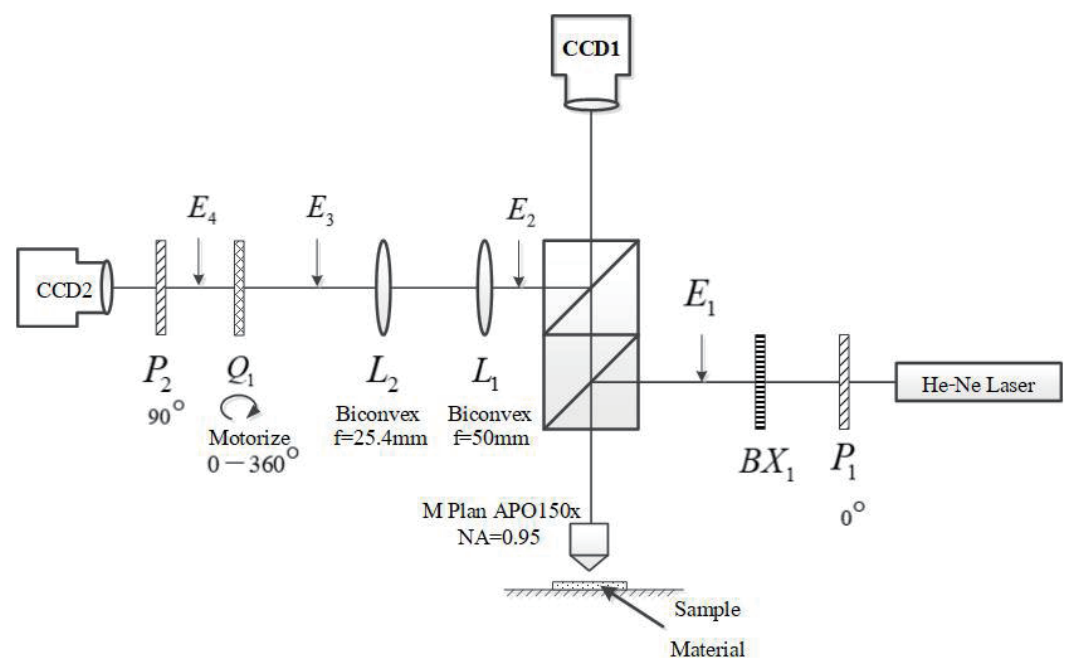

Fig. 2. Diagram of back focal plane micro-ellipsometer.

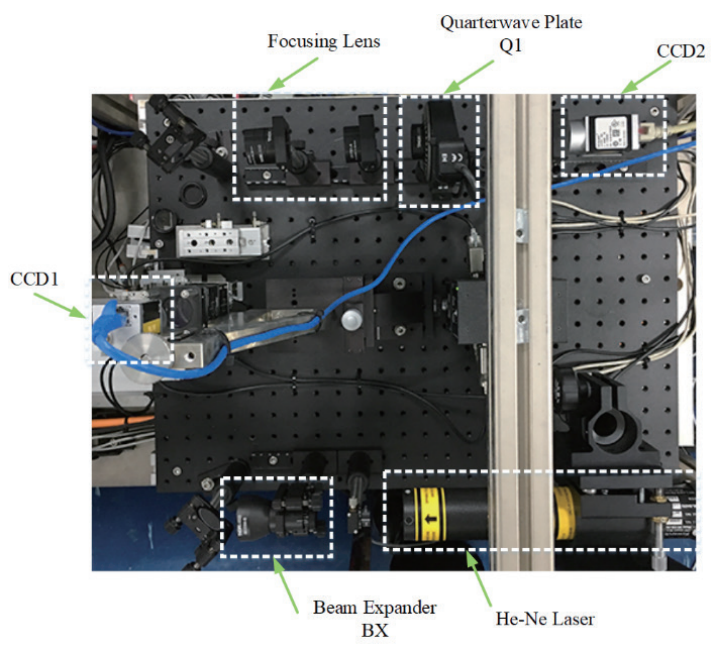

(a)

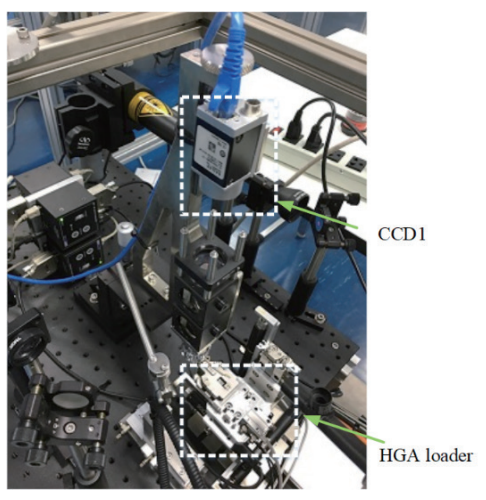

(b)

Fig. 3. (Color online) Optical system for flying height measurements. (a) Full diagram of micro-ellipsometer. (b) HGA loader. 
objective lens. The magnification of the objective lens is $150 \times$; therefore, the spot size of the beam is less than $0.1 \mu \mathrm{m}$. Such a small spot size is beneficial for performing the measurement on the relatively small recording head (typical measurement area of $50 \times 50 \mu \mathrm{m}^{2}$ ). The NA of the objective lens is 0.95 and its incident angle is $0-71.8051^{\circ}$, which is derived from

$$
N A=n \sin (\theta)
$$

After the beam passes through the objective lens, the light is reflected back from the material to the objective lens and passes through the beam splitters. After passing the second beam splitter, the reflected beam is transmitted to the $\mathrm{L}_{1}$ and $\mathrm{L}_{2}$ lenses. The positions of $\mathrm{L}_{1}$ and $L_{2}$ lenses are fixed so that the reflected beam at point $E_{3}$ is the image of the back-focal plane of the objective lens. The reflected beam is then directed to QWP $\mathrm{Q}_{1}$ and polarizer $\mathrm{P}_{2}$, respectively. In the last step, the CCD2 camera is used to capture an image of the beam passing through polarizer $\mathrm{P}_{2}$. QWP $\mathrm{Q}_{1}$ is rotated from 1 to 360 with an azimuthal angle resolution of 1 degree per step. The CCD2 camera is then used to record an image for each azimuthal rotation step. The typical recorded images for azimuthal angles of $0,45,90$, and $135^{\circ}$ are shown in Fig. 4.

\subsection{Image analysis procedure for retrieving $n, k$ values from recorded images}

The flowchart for the image analysis procedure for recovering $n, k$ values from the recorded images is shown in Fig. 5. The first step in optical image analysis begins with the images captured by the CCD2 camera. The color image is initially converted to a grayscale and QWP $\mathrm{Q}_{1}$ is rotated from 1 to $360^{\circ}$ with the resolution of $1^{\circ}$. Therefore, in one measurement circle, there are 360 recorded images. The typical recorded image is shown in Fig. 6. For each recorded image, the intensity level data point is selected along the line at 45 diagonal as shown in Fig. 6. This selected data is called a data line for each recorded image. In the next process, each data line for each recording image is stacked in column-vise. The resulting image from the stacking of $\mathrm{SiO}_{2}$ measurement images is shown in Fig. 7. This image is called the stacked intensity image (SII). In order to retrieve the optical phase shift value for each incident angle, the fast Fourier transform (FFT) is then performed on each horizontal data line of SII. The phase shift values of FFT calculation for each incident angle are plotted. The resulting plot, the

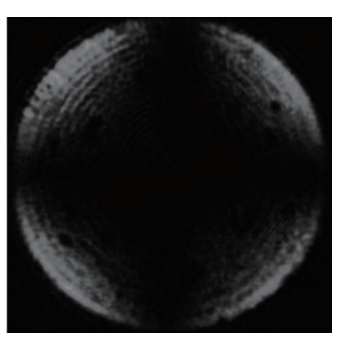

(a)

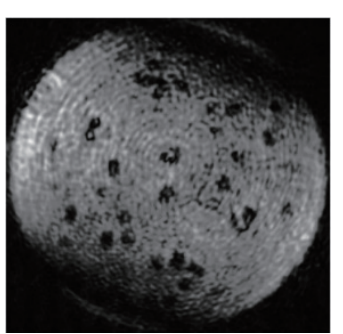

(b)

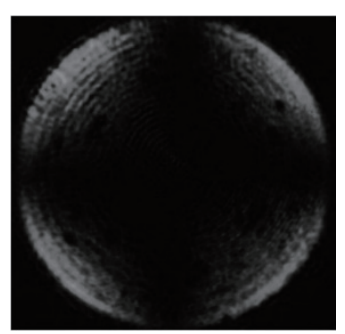

(c)

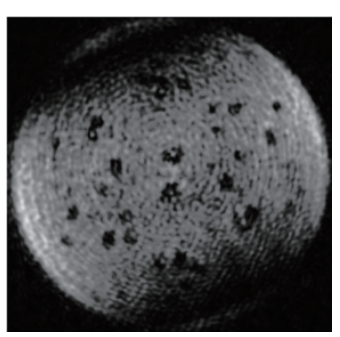

(d)

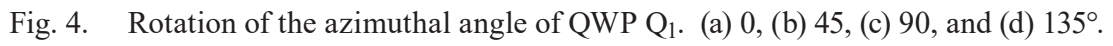




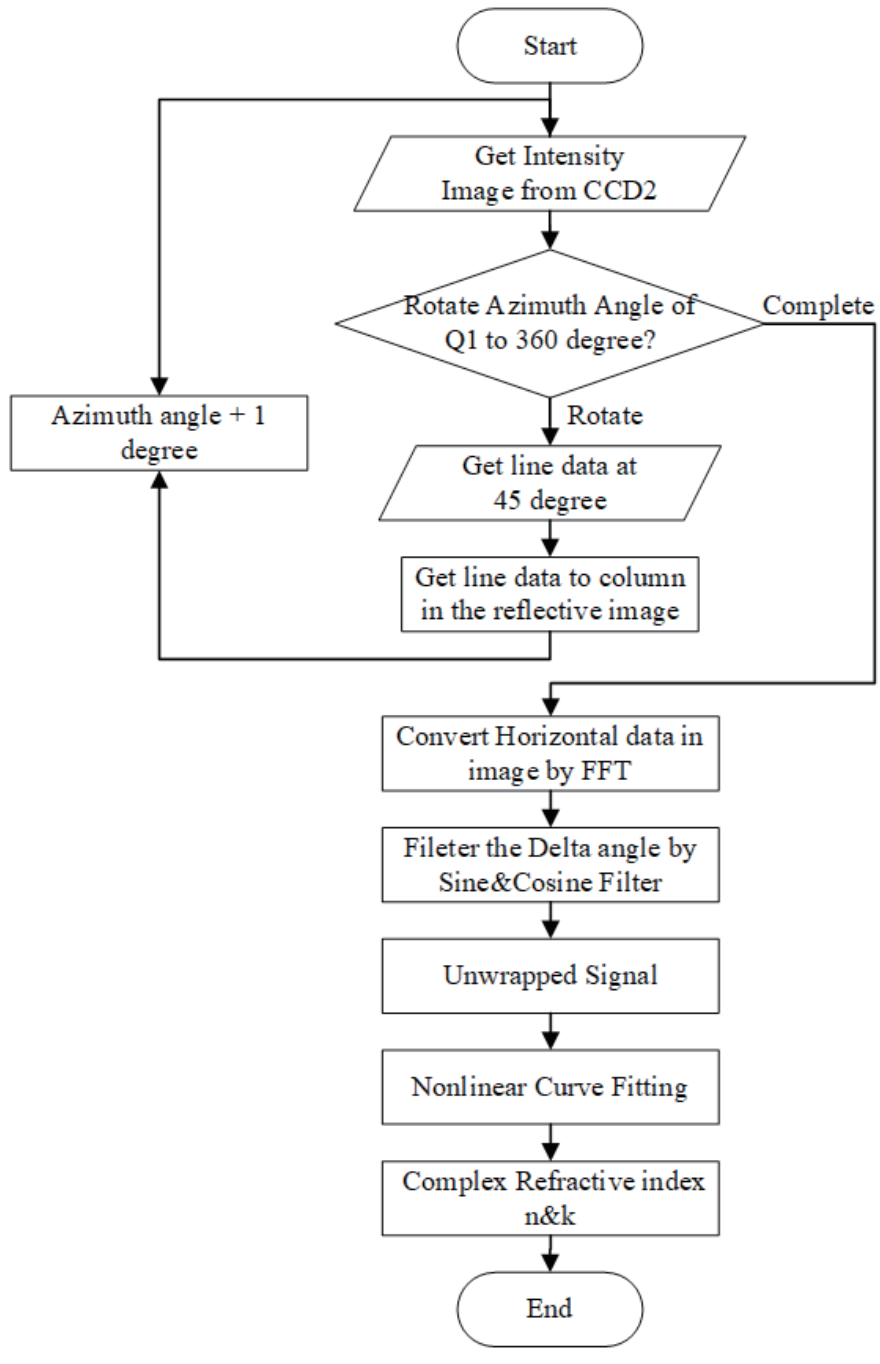

Fig. 5. Procedure of optical image processing.

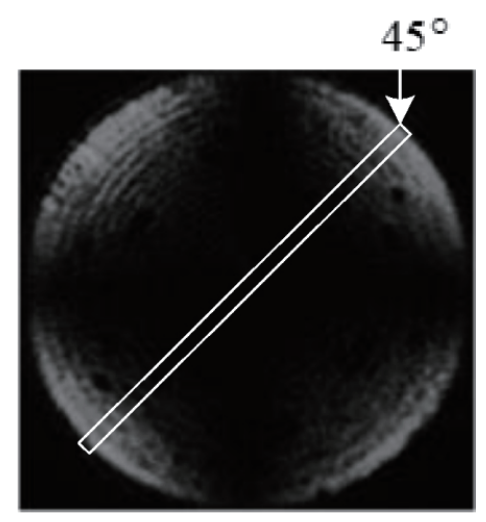

Fig. 6. Intensity image of $\mathrm{Q}_{1}\left(\right.$ azimuth $\left.=1^{\circ}\right)$.

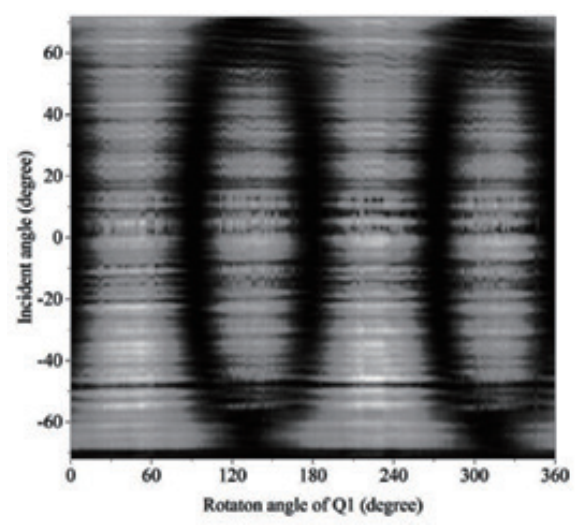

Fig. 7. Stack intensity images of $\mathrm{SiO}_{2}$ materials.

so-called delta angle, is shown in Fig. 8. The delta angle is unwrapped to eliminate the transient impulse phase changes. The $n, k$ value is then determined by the curve fitting procedure for the measured delta angle.

\subsection{Distribution of measured $n, k$ value from a micro-ellipsometer system with a MQTT protocol}

In our system, we choose the MQTT protocol to transfer the measured $n, k$ values to the cyber space. The measured data are transmitted to subscribers such as flying height tester engineers, managers, and designers in headquarter, or setting up engineers for a flying height tester (D6). The flying height tester engineer can use the data for analysis while headquarters can use the data to report the HDD quality. Figure 9 shows the diagram of the configuration of a private cloud measurement system. 


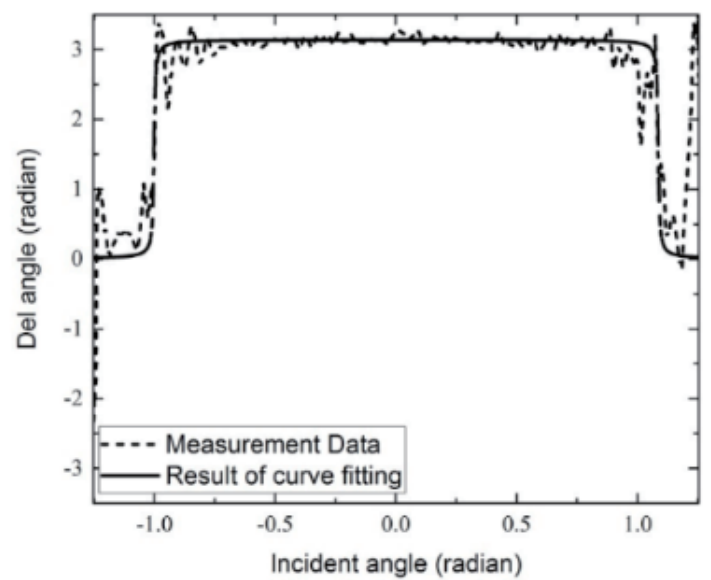

Fig. 8. Delta angle of $\mathrm{SiO}_{2}$.

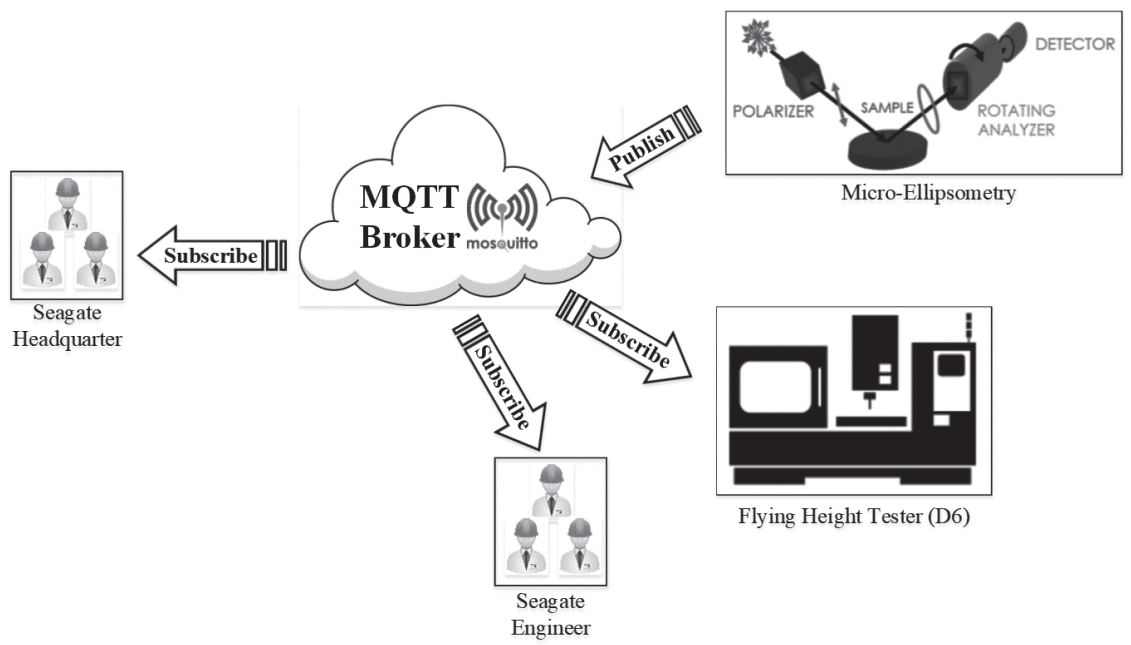

Fig. 9. Private cloud structure of optical characterization.

However, the correct receiving order of the measured reflective values is important for the user requirement in order to trace measurement data for every recording head. Therefore, we need to consider the QoS levels so that it is sufficient for both message delivery and latency for data communication. The MQTT push protocol, which is a settlement between the sender and receiver of a single message regarding the guarantee of message delivery, normally describes the transmission QoS levels. There are three transmission QoS levels in MQTT.

- QoS 0: At most once. A message is sent at most once and the server does not guarantee of delivering a message.

- QoS 1: At least once. A message is sent at least once and it is possible to deliver a message more than once.

- QoS 2: Exactly once. A message is sent exactly once with 4-way handshaking.

In our experiment, we perform the test on the data communication via the MQTT protocol for different QoS levels. The measured round-trip time and throughput are recorded for each QoS level. 
The Mosquitto MQTT server is configured for private cloud data communication. The inhouse communication program embedded in the micro-ellipsometer system is developed using Microsoft visual C\# and Node-Red. The program reformats the measured $n, k$ values to be in a JSON format and send to the private cloud network via MQTT. The Node-Red server receives data and splits them into $n, k$ values as shown in Fig. 10. The $n, k$ data are plotted in separate graphs. The stakeholder can monitor data through web interface service.

\section{Results and Discussion}

\subsection{Micro-ellipsometer measurement results}

Figure 11 shows the SIIs of glass $\left(\mathrm{SiO}_{2}\right)$, gold $(\mathrm{Au})$, and aluminium titanium carbon (AlTiC). The intensity patterns of SIIs evidently vary depending on the optical properties of materials. Each intensity pattern is in a bright-dark format, which comprises 4 repetitive cycles. Therefore, in order to extract the phase shift for each incident angle, we need to perform the FFT calculation and select only the phase shift of the fourth-order harmonic to create the delta angle for each incident angle as shown in Fig. 12. The $x$-axis is the incident angle and the $y$-axis is the delta angle (optical phase shift). The incident angle ranges from $\pi$ to $-\pi$. The delta angle is therefore filtered by sine and cosine filters. The sine and cosine filters are normally used to eliminate spike phase shift noise. In the final process, the complex refractive index $(n, k)$ is determined by nonlinear curve fitting to the delta angle graph using the least-squares method (LSM).

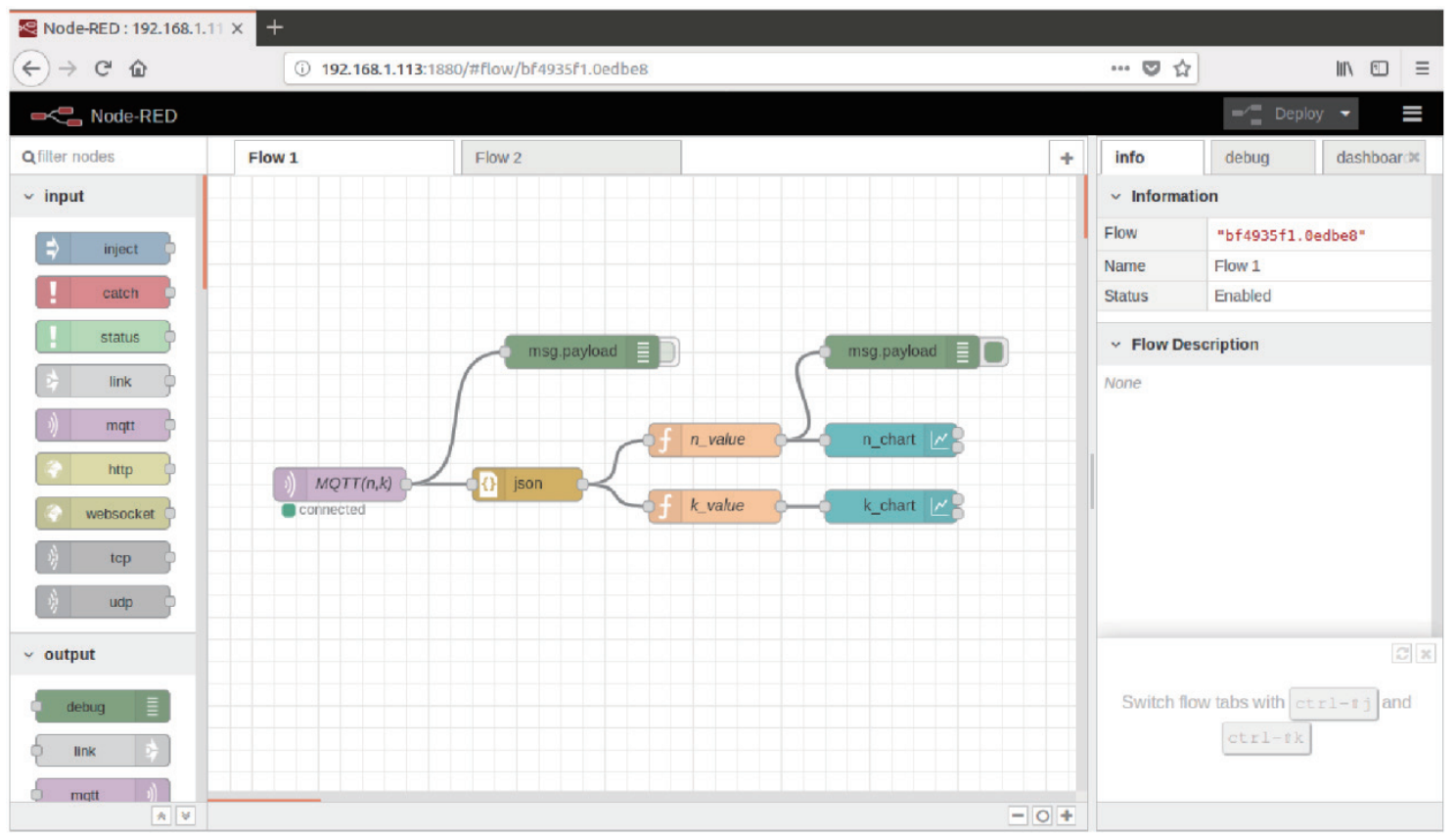

Fig. 10. (Color online) Workflow of Node-Red. 


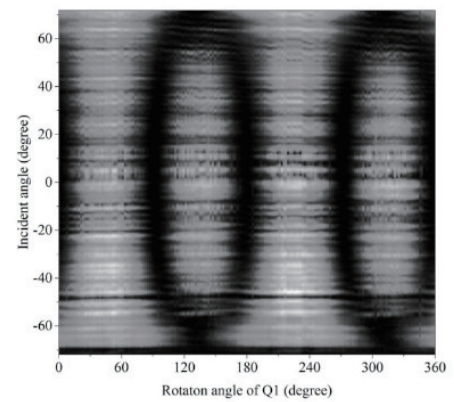

(a)

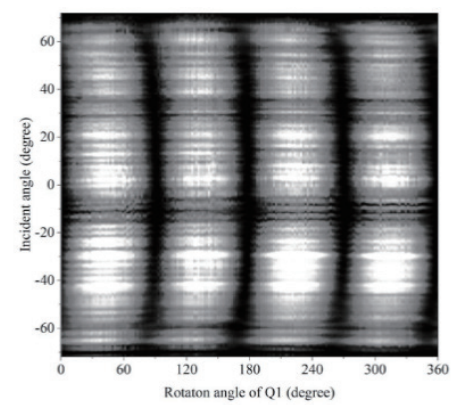

(b)

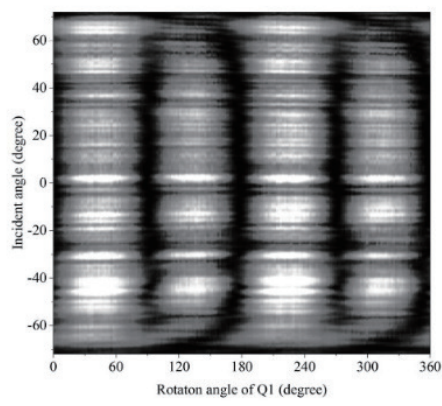

(c)

Fig. 11. Stack intensity images of (a) $\mathrm{SiO}_{2}$, (b) Au, and (c) AlTiC.

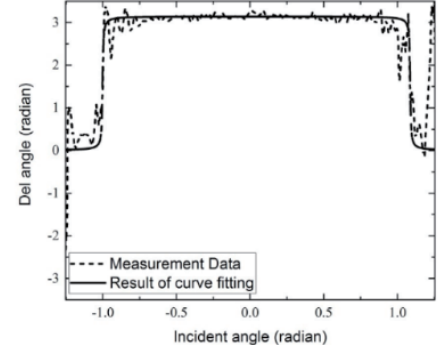

(a)

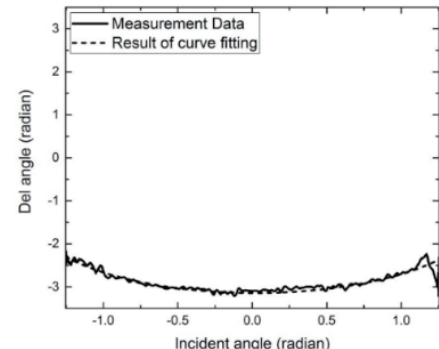

(b)

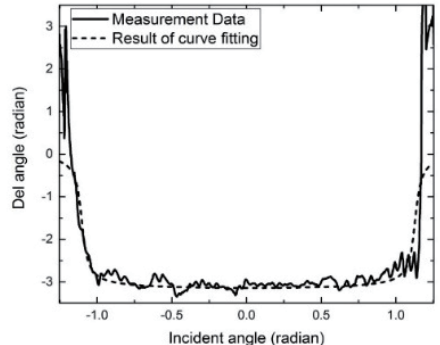

(c)

Fig. 12. Delta angles of (a) $\mathrm{SiO}_{2}$, (b) Au, and (c) AlTiC.

Table 1 shows the reference and measured values. The complex refractive index consists of the refractive index $(n)$ and extinction coefficient $(k)$ (http://www.filmetrics.com). The measured $n, k$ values show good correlation twith their reference values.

\subsection{Distribution of $n, k$ values via MQTT results}

For the distribution of the measured $n, k$ values from the micro-ellipsometer, the data is sent to the cloud network by MQTT according to the system described in the previous section. Figure 13 shows the GUI interface for monitoring $n, k$ values for all users. We choose a standard IEEE 802.3 wireline network to communicate instead of the standard IEEE 802.11 wireless network. This is due to the fact that the performance of the wireless network is reduced by a large number of clients and the frequency and channel interference. The micro-ellipsometer has an operating cycle time of $1 \mathrm{~min}$. Our MQTT system transmits and receives data for each cycle typically less than $1 \mathrm{~min}$ as shown in Fig. 14. The QoS level setting shows a significant effect on the round-trip time as expected (Table 2). However, the communication time using MQTT is greatly less than the operating cycle of the micro-ellipsometer; thus, we are assured that the measurement data transmitted through the network is certainly in the correct recieving order. Figure 15 and Table 3 show a summary of the throughputs of the MQTT system in different QoS setting. 
Table 1

$n, k$ values of practical standards and measurements.

\begin{tabular}{llcc}
\hline & Refractive index & Refractive index $(n)$ & Extinction coefficient $(k)$ \\
\hline \multirow{2}{*}{$\mathrm{SiO}_{2}$} & Practical standard & 1.460 & 0.000 \\
& Measurement value & 1.519 & -0.010 \\
\hline \multirow{2}{*}{$\mathrm{Au}$} & Practical standard & 0.181 & 3.068 \\
& Measurement value & 0.136 & 4.133 \\
\hline \multirow{2}{*}{ AlTiC } & Practical standard & 2.236 & 0.426 \\
& Measurement value & 2.295 & 0.200 \\
\hline
\end{tabular}

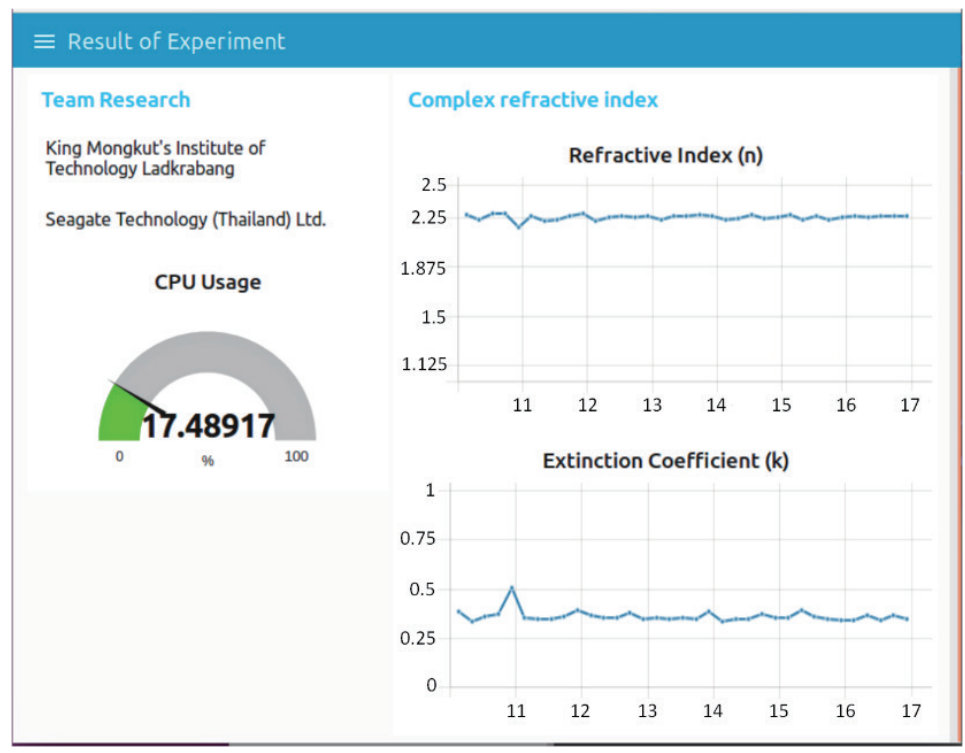

Fig. 13. (Color online) Experimental results.

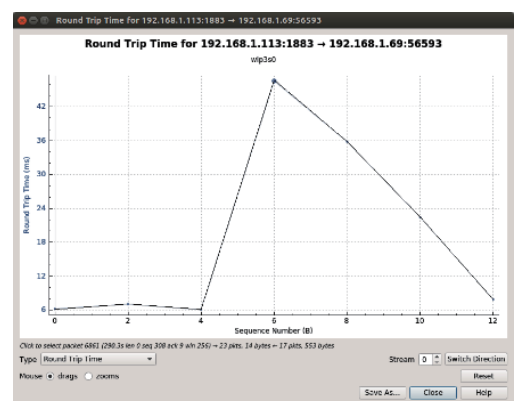

(a)

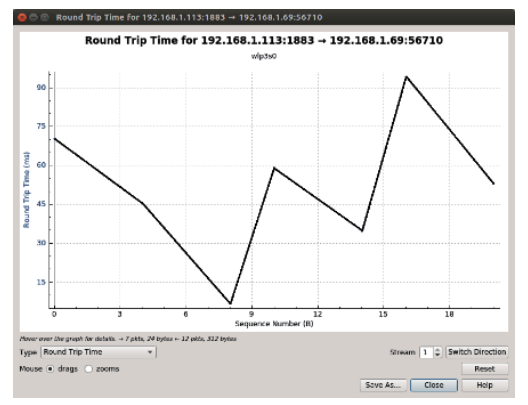

(b)

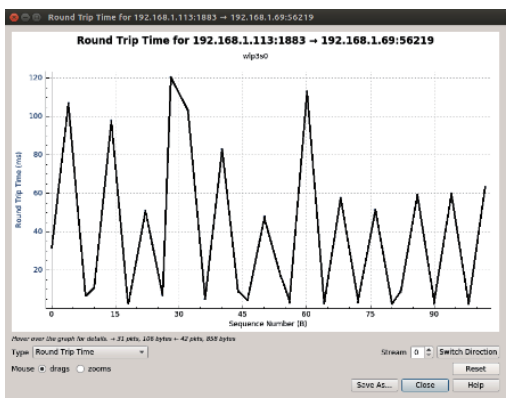

(c)

Fig. 14. (Color online) Round-trip time of MQTT communication.

Table 2

Round-trip time averages.

\begin{tabular}{lccc}
\hline & QoS $=0$ (At most once) & QoS $=1$ (At least once) & QoS = 2 (Exactly once) \\
\hline Round-trip time average & $18.714 \mathrm{~ms}$ & $51.429 \mathrm{~ms}$ & $39.107 \mathrm{~ms}$ \\
\hline
\end{tabular}




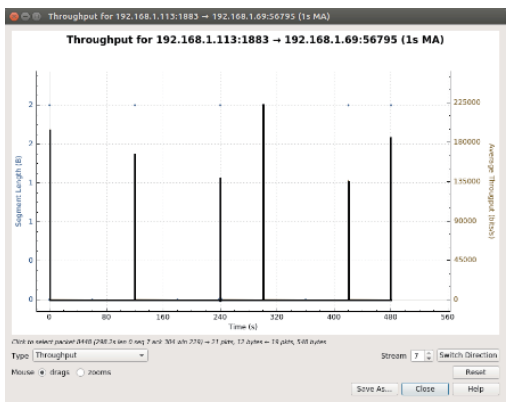

(a)

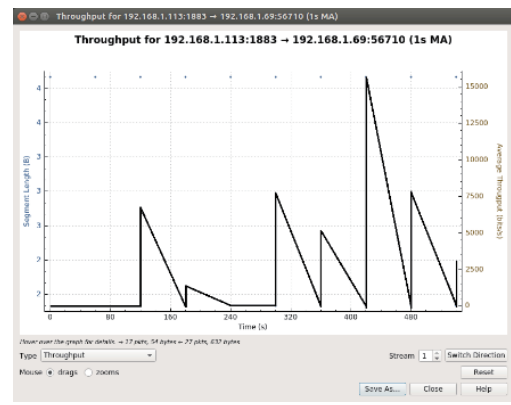

(b)

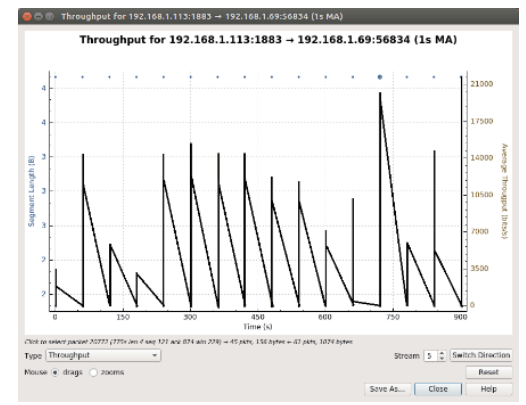

(c)

Fig. 15. (Color online) Throughput of MQTT communication.

Table 3

Maximum throughput of MQTT communication.

\begin{tabular}{lccc}
\hline & QoS $=0$ (At most once) & QoS $=1$ (At least once) & QoS = 2 (Exactly once) \\
\hline Maximum throughput & $225000 \mathrm{bps}$ & $15500 \mathrm{bps}$ & $21700 \mathrm{bps}$ \\
\hline
\end{tabular}

\section{Conclusions}

In this paper, a cloud-based measurement system for recording head optical characterization is presented. The system can be used to determine the complex reflective index $(n, k)$. The measured $n, k$ values are transmitted over the private cloud network using the MQTT protocol. These optical constants are sent to the stakeholders and the flying height machine (D6). The micro-ellipsometer utilizes standard samples of $\mathrm{Au}$ and $\mathrm{SiO}_{2}$ for calibration. The results of measurements are close to the range of reference values and industrial standard. This method of flying height measurement improves the measurement speed with smaller spot size for individual recording heads and also the sharing measurement data between the stakeholders. In addition, the round-trip time of MQTT data communication shows that the communication time using MQTT is greatly less than the operating cycle of the micro-ellipsometer. With these results, we are assured that the measurement data transmitted through the network is certainly in the correct receiving order.

\section{Acknowledgments}

This work was supported by Seagate Technology (Thailand), King Mongkut's Institute of Technology Ladkrabang and Rajamangala University of Technology Phra Nakhon.

\section{References}

1 S. Yammen and P. Muneesawang: IEEE Trans. Compon. Packag. Manuf. Technol. 4 (2014) 1523.

2 S. H. Leong, Z. M. Yuan, K. W. Ng, and B. Liu: IEEE Trans. Mag. 42 (2006) 2534.

3 G. D. Feke, D. P. Snow, R. D. Grober, P. J. de Groot, and L. Deck: Appl. Opt. 37 (1998) 1796.

4 C. Ai and J. C. Wyant: Appl. Opt. 26 (1987) 1112. 
5 K. Creath: Temporal Phase Measurement Methods (Wiley, New York, 1993).

6 H.-K. Teng and K.-C. Lang: Opt. Lasers Eng. 46 (2008) 203.

7 D. C. Y. Vargas and C. E. P. Salvador: IEEE Lat. Am. Trans. 14 (2016) 3900.

8 N. Tantitharanukul, K. Osathanunkul, K. Hantrakul, P. Pramokchon, and P. Khoenkaw: Int. Conf. Digital Arts, Media and Technology (ICDAMT) (2017) 62.

9 IBM MQTT Protocol Specification: http://public.dhe.ibm.com/software/dw/webservices/ws-mqtt/mqtt-v3rl. html (accessed December 2015).

10 S. Lee, I. Jung, H. Kim, and H. Ju: Asia-Pacific Network Operations and Management Symp. (APNOMS) (2013) 1. 